\title{
Transcutaneous vagus nerve stimulation (tVNS) modulates flow experience
}

\author{
Lorenza S. Colzato ${ }^{1,2,3,5}$ - Gina Wolters ${ }^{4}$ Corinna Peifer $^{4}$
}

Received: 1 August 2017 / Accepted: 6 November 2017 / Published online: 11 November 2017

(c) The Author(s) 2017. This article is an open access publication

\begin{abstract}
Flow has been defined as a pleasant psychological state that people experience when completely absorbed in an activity. Previous correlative evidence showed that the vagal tone (as indexed by heart rate variability) is a reliable marker of flow. So far, it has not yet been demonstrated that the vagus nerve plays a causal role in flow. To explore this we used transcutaneous vagus nerve stimulation (tVNS), a novel non-invasive brain stimulation technique that increases activation of the locus coeruleus (LC) and norepinephrine release. A sham/placebo-controlled, randomized cross-over within-subject design was employed to infer a causal relation between the stimulated vagus nerve and flow as measured using the Flow Short-Scale in 32 healthy young volunteers. In both sessions, while being stimulated, participants had to rate their flow experience after having performed a task for $30 \mathrm{~min}$. Active tVNS, compared to sham stimulation, decreased flow (as indexed by absorption scores). The results can be explained by the network reset theory, which assumes that high-phasic LC activity promotes a global reset
\end{abstract}

Lorenza S. Colzato

colzato@fsw.leidenuniv.nl

1 Cognitive Psychology Unit and Leiden Institute for Brain and Cognition, Leiden University, Leiden, The Netherlands

2 Department of Cognitive Psychology, Institute of Cognitive Neuroscience, Faculty of Psychology, Ruhr University Bochum, Bochum, Germany

3 Institute for Sports and Sport Science, University of Kassel, Kassel, Germany

4 Unit Applied Psychology in Work, Health, and Development, Faculty of Psychology, Ruhr University Bochum, Bochum, Germany

5 Cognitive Psychology Unit, Leiden University Institute for Psychological Research, Wassenaarseweg 52, 2333 AK Leiden, The Netherlands of attention over exploitation of the current focus of attention, allowing rapid behavioral adaptation and resulting in decreased absorption scores. Furthermore, our findings corroborate the hypothesis that the vagus nerve and noradrenergic system are causally involved in flow.

Keywords Transcutaneous vagus nerve stimulation . Locus coeruleus $\cdot$ Norepinephrine $\cdot$ Flow experience . Network reset theory $\cdot$ Parasympathetic activation

\section{Introduction}

The subjective experience of flow has been defined as a pleasant psychological state that people experience when completely absorbed in an activity (Csikszentmihalyi 1975). Flow has been described as a rise of concentration and attention, and enhanced sense of control without keeping track of time (Csikszentmihalyi 1975, 1991). Only recently have the first studies investigating the physiology of flow been published (de Manzano et al. 2010; Keller et al. 2011; for an overview see; Peifer 2012; Peifer et al. 2014, 2015), and they suggest flow experience to be associated with moderate activation of the sympathetic nervous system and hypothalamus-pituitary-adrenal axis. Further, studies have shown the involvement of parasympathetic activation (i.e., vagal tone) in modulating flow (Peifer et al. 2014; Tozman et al. 2015), thus pointing to a crucial role of the vagus nerve. Unfortunately, the nature of the studies mentioned above is correlative and, so far, it has not yet been demonstrated that the vagus nerve plays a causal role in flow. The vagus nerve is the tenth cranial nerve and represents a key component of the parasympathetic nervous system. Notably, the vagus nerve is the longest cranial nerve and has the largest 
distribution in the body. It includes somatic and visceral afferent fibers, as well as general and special visceral efferent fibers.

Given the correlational nature of previous studies, the aim of the current study is to examine the causal involvement of the vagus nerve in the subjective experience of flow. To do that, we used transcutaneous (through the skin) vagus nerve stimulation (tVNS), a novel non-invasive brain stimulation technique proposed for the first time by Ventureyra (2000). In contrast to correlational techniques, with tVNS we are able to infer a causal relation between the stimulated vagus nerve and flow as indexed by the Flow Short-Scale (Engeser and Rheinberg 2008).

tVNS activates the auricular branch of the vagus nerve $(\mathrm{ABVN})$, which innervates the skin of the concha in the human ear (Peuker and Filler 2002) and allows for reliable transcutaneous electrical stimulation of the nerve fibers in this area. In an influential study, Fallgatter et al. (2003) stimulated the tragus and showed that active tVNS, compared to sham, produced a clear and reliable vagus sensory evoked potential in healthy participants. Further, two functional magnetic resonance imaging (MRI) studies in healthy humans have found that tVNS increased activation in the brainstem region including the locus coeruleus (LC) and nucleus of the solitary tract, indicating that tVNS is able to effectively stimulate vagal afferents to the brainstem (Dietrich et al. 2008; Frangos et al. 2015). In rats, it has been shown that VNS leads to an intensity-dependent increase in brain norepinephrine (NE) in response to stimulation of the left vagus nerve (Raedt et al. 2011; Roosevelt et al. 2006). These increases in NE are transient and return to baseline levels when the stimulation is terminated and the vagus nerve is no longer being activated (Roosevelt et al. 2006). The fact that active tVNS increases the activation of LC and NE release has important implications for the investigation of the biological underpinning of flow.

As described, flow experience is characterized by high concentration and attention to the task at hand (Csikszentmihalyi 1975). The network reset theory proposes that phasic LC activity promotes a global reset of attention over exploitation of the current focus of attention allowing rapid behavioral adaptation (Bouret and Sara 2005). If this is the case, phasic increase in LC activity should decrease focused attention resulting in decreased subjective experience of flow.

In sum, the goal of the current study was to test if the vagus nerve is causally involved in the experience of flow via its role in activating the $\mathrm{LC}$ and increasing NE release. Therefore, while being stimulated, participants had to rate their flow experience after having performed a behavioral task. Following the network reset theory (Bouret and Sara 2005), the increase of LC activity is expected to reduce focused attention resulting in decreased flow.

\section{Experimental procedures}

\section{Participants}

Thirty-two Leiden University undergraduate students (22 females, 10 males, mean age $=21.34$ years, range $18-28$ ) took part in the study. Participants were enrolled via an online recruiting system and were given either course credits or a financial reward of 10 euros for participating in a study on the effects of brain stimulation on decision-making. Once recruited, all participants were screened individually by the same lab-assistant using the Mini International Neuropsychiatric Interview (M.I.N.I.; Sheehan et al. 1998). The M.I.N.I. is a short, structured interview of about $15 \mathrm{~min}$ that screens for several psychiatric disorders and drug use, and it is often used in clinical and pharmacological research (Colzato et al. 2012, 2013). Following previous published protocols (Colzato et al. 2017; Sellaro et al. 2015; Steenbergen et al. 2015; Beste et al. 2016) participants took part in the experiment only if they met the following criteria: (1) age between 18 and 30 years; (2) no history of neurological or psychiatric disorders; (3) no history of substance abuse or dependence; (4) no history of brain surgery, tumors, or intracranial metal implantation; (5) no chronic or acute medications; (6) no pregnancy; (7) no susceptibility to seizures or migraine; (8) no pacemaker or other implanted devices. All participants never experienced tVNS before this study. Before the beginning of the testing session, they were given a verbal and written description of the procedure and of the usual adverse effects (i.e., itching and tingling skin sensation, skin-reddening, and headache). Participants received no information about the different types of stimulation (active vs. sham) or about the assumptions regarding the study. The experiment conformed to the ethical standards of the Declaration of Helsinki and the protocol was approved by the local ethical committee (Leiden University, Institute for Psychological Research). Written informed consent was obtained from all participants.

\section{Apparatus and procedure}

A single-blind, sham/placebo-controlled, randomized crossover within-subject design with counterbalanced order of conditions was used to assess the effect of online (i.e., stimulation overlapping with the critical task) tVNS on flow performance in healthy young volunteers.

All participants took part in two sessions (active vs. sham), separated by 1 week, and were tested individually. Twenty minutes after the onset of stimulation, participants performed for $30 \mathrm{~min}$ an emotion recognition task that requires participants to assess someone's emotions based on images of whole faces and bodies. Participants were asked to choose which of four emotions (i.e., happy, 
fear, anger, and sad) better described what the person in the image was feeling. The four emotional labels were displayed at the four corners of an imagined square surrounding the target picture, and participants had to click with the computer mouse on the chosen emotion. Because of technical failure, the data of the emotion recognition task have not been further analyzed or published elsewhere. After task completion, participants had to rate their flow experience on the Flow Short-Scale (Engeser and Rheinberg 2008). Afterwards, the stimulation was terminated and participants were asked to complete a tVNS adverse effects questionnaire requiring them to rate, on a five-point (1-5) scale, how much they experienced (1) headache, (2) neck pain, (3) nausea, (4) muscle contraction in face and/or neck, (5) stinging sensation under the electrodes, (6) burning sensation under the electrodes, (7) uncomfortable (generic) feelings, and (8) other sensations and/or adverse effects. None of the participants reported major complaints or discomfort during or after tVNS. They were explicitly asked if they could guess the stimulation received and no one reported to be aware of it.

\section{Transcutaneous vagus nerve stimulation (tVNS)}

We employed the NEMOS ${ }^{\circledR}$ tVNS neurostimulating device. This device is composed by a stimulation unit and a dedicated ear electrode, which can be worn like an earphone. Following previous published protocols for optimal stimulation (Colzato et al. 2017; Sellaro et al. 2015; Steenbergen et al. 2015; Beste et al. 2016), the $\mathrm{tVNS}^{\circledR}$ device was programmed to a stimulus intensity at $0.5 \mathrm{~mA}$, delivered with a pulse width of $200-300 \mu \mathrm{s}$ at $25 \mathrm{~Hz}$. Stimulation alternated between on and off periods every $30 \mathrm{~s}$. In the active condition, the stimulation electrodes were applied to the outer auditory canal. In the sham (placebo) condition, the stimulation electrodes were placed on the center of the left ear lobe. Indeed, the ear lobe has been found to be free of cutaneous vagal innervation (Peuker and Filler 2002; Fallgatter et al. 2003) and a recent fMRI study found that this sham condition produced no activation in the cortex and brain stem (Kraus et al. 2013).

Further, following safety criteria to avoid cardiac side effects, the stimulation was always applied to the left ear (Nemeroff et al. 2006; Cristancho et al. 2011). Indeed, although efferent fibers of the vagus nerve affect cardiac function, such an impact seems to relate only to the efferent vagal fibers connected to the right ear (Nemeroff et al. 2006). Consistent with this picture, a clinical trial reported no arrhythmic effects of tVNS when applied to the left ear (Kreuzer et al. 2012).

\section{Flow Short-Scale}

Flow experience was indexed by the two subscales absorption and fluency of the Flow Short-Scale (Engeser and Rheinberg 2008). The items are assessed on a seven-point Likert scale from 1 (I don't agree) to 7 (I agree). Absorption consists of four items (e.g., "I do not recognize that time is going by") and fluency is measured by six items (e.g., "I feel that everything is under control"). We found good to satisfactory reliabilities of absorption (Cronbach's $\alpha=0.65$ ) and fluency (Cronbach's $\alpha=0.73$ ) replicating previous protocols (Peifer et al. 2014). Participants filled in the Flow ShortScale immediately after they finished the emotion recognition task and were instructed to refer to it when answering the questionnaire.

\section{Statistical analyses}

For each participant, and for both the active and the sham stimulation, the scores for the absorption and fluency subscales were calculated. To examine whether active tVNS, as compared to sham (placebo) stimulation, affected flow experience, two separate repeated measures analysis of variance (ANOVA) were carried out with absorption and fluency as dependent variables and session (active vs. sham) as withinparticipant factor.

A significance level of $p<0.05$ was adopted for all statistical tests.

\section{Results}

\section{Absorption}

Repeated measures ANOVA revealed a significant main effect of session on absorption scores, $F(1,31)=7.34$, $p<0.05, \eta 2 p=0.191$ : participants showed decreased scores in the active (3.97, SEM =1.56) compared to the sham (4.36, $\mathrm{SEM}=1.41)$ session.

\section{Fluency}

Repeated measures ANOVA revealed no significant main effect of session on fluency scores, $F(1,31)=1.46, p=0.24$, $\eta 2 p=0.045$ : participants showed comparable scores in the sham $(5.21, \mathrm{SEM}=1.40)$ and in the active $(4.99$, $\mathrm{SEM}=1.54$ ) session.

\section{Discussion}

Our findings show that tVNS, likely via activation of LC and increased NE release, modulates flow. Indeed, when 
actively stimulated, participants show decreased flow (as indexed by absorption scores). The observation that tVNS modulates ratings on a well-established diagnostic index of flow (Engeser and Rheinberg 2008) provides considerable support for the idea of a crucial and causal role of noradrenergic pathways in flow. As absorption scores decreased by means of tVNS, our results are in line with the network reset theory (Bouret and Sara 2005) which assumes that high-phasic LC activity promotes a global reset of attention over exploitation of the current focus of attention allowing rapid behavioral adaptation and resulting in decreased absorption scores.

Given that none of the participants reported major complaints or discomfort during or after the active and sham condition and that none could guess the stimulation received, we can rule out an explanation of our results in these terms. This is not to deny, however, that more research is needed to support this conclusion. Nonetheless, given the causal nature of the brain stimulation technique used in the current study, our findings provide a first direct demonstration for a causal link between the vagus nerve and the subjective experience of flow. Although stimulation of the vagus is, besides NE, also associated with increased GABA release (Ben-Menachem et al. 1995; Marrosu et al. 2003), to the best of our knowledge there is no indication in the literature that changes in GABA might have been responsible for our results.

Our results are in line with previous correlative studies showing that heart rate variability (an index of vagal tone) was associated with flow experience (Peifer et al. 2014; Tozman et al. 2015). Future studies should replicate our findings and include a variation of the task demands before measuring the experience of flow. This would be important because task demands seem to play a role in modulating the direction of the subjective experience of flow (Peifer et al. 2014, 2017; Tozman et al. 2015).

Finally, there are some limitations of the current study that need to be outlined. First, it would have been optimal to have combined the application of tVNS with suitable physiological assays, such as vagus somatosensory evoked potentials (Hagen et al. 2014, 2015). Moreover, it would be useful to include pupil dilation measures, a physiological correlate of LC-NE activity (Murphy et al. 2014).

Notwithstanding these limitations, our findings provide direct evidence for the idea that the vagus nerve plays a causal role in flow, via its role in activating the LC and increasing NE release. Hence, these observations may stimulate research to further extend our understanding of the specific role of the vagus nerve, LC and NE in the general experience of flow. Further, our results support the idea that tVNS is a promising non-invasive brain stimulation technique for modulating mental processes in healthy humans (van Leusden et al. 2015).
Open Access This article is distributed under the terms of the Creative Commons Attribution 4.0 International License (http://creativecommons.org/licenses/by/4.0/), which permits unrestricted use, distribution, and reproduction in any medium, provided you give appropriate credit to the original author(s) and the source, provide a link to the Creative Commons license, and indicate if changes were made.

\section{References}

Ben-Menachem E, Hamberger A, Hedner T, Hammond EJ, Uthman BM, Slater J, Wilder BJ (1995) Effects of vagus nerve stimulation on amino acids and other metabolites in the CSF of patients with partial seizures. Epilepsy Res 20:221-227

Beste C, Steenbergen L, Sellaro R, Grigoriadou S, Zhang R, Chmielewski W, Stock A-K, Colzato L (2016) Effects of concomitant stimulation of the GABAergic and norepinephrine system on inhibitory control-a study using transcutaneous vagus nerve stimulation. Brain Stimul 9(6):811-818

Bouret S, Sara SJ (2005) Network reset: a simplified overarching theory of locus coeruleus noradrenaline function. Trends Neurosci 28(11):574-582

Colzato LS, de Bruijn ER, Hommel B (2012) Up to "Me" or Up to "Us"? The impact of self-construal priming on cognitive selfother integration. Front Psychol 3:341. https://doi.org/10.3389/ fpsyg.2012.00341

Colzato LS, van den Wildenberg WP, Hommel B (2013) The genetic impact (C957T-DRD2) on inhibitory control is magnified by aging. Neuropsychologia 51:1377-1381

Colzato LS, Sellaro R, Beste C (2017) Darwin revisited: the vagus nerve is a causal element in controlling recognition of other's emotions. Cortex 92:95-102

Cristancho P, Cristancho MA, Baltuch GH, Thase ME, O'Reardon JP (2011) Effectiveness and safety of vagus nerve stimulation for severe treatment-resistant major depression in clinical practice after FDA approval: outcomes at 1 year. J Clin Psychiatry 72:1376-1382

Csikszentmihalyi M (1975) Beyond boredom and anxiety. JosseyBass Publishers, San Francisco

Csikszentmihalyi M (1991) Flow: the psychology of optimal experience. Harper \& Row, New York

de Manzano Ö, Theorell T, Harmat L, Ullén F (2010) The psychophysiology of flow during piano playing. Emotion 10(3):301-311

Dietrich S, Smith J, Scherzinger C, Hofmann-Preiß K, Freitag T, Eisenkolb A, Ringler R (2008) A novel transcutaneous vagus nerve stimulation leads to brainstem and cerebral activations measured by functional MRI/Funktionelle Magnetresonanztomographie zeigt Aktivierungen des Hirnstamms und weiterer zerebraler Strukturen unter transkutaner Vagusnervstimulation. Biomedizinische Technik/Biomed Eng 53(3):104-111

Engeser S, Rheinberg F (2008) Flow, performance and moderators of challenge-skill balance. Motivation Emot 32(3):158-172

Fallgatter AJ, Neuhauser B, Herrmann MJ, Ehlis AC, Wagener A, Scheuerpflug P, Riederer P (2003) Far field potentials from the brain stem after transcutaneous vagus nerve stimulation. J Neural Transm 110(12):1437-1443

Frangos E, Ellrich J, Komisaruk BR (2015) Non-invasive access to the vagus nerve central projections via electrical stimulation of the external ear: fMRI evidence in humans. Brain Stimul $8(3): 624-636$

Hagen K, Ehlis A-C, Schneider S, Haeussinger FB, Fallgatter AJ, Metzger FG (2014) Influence of different stimulation parameters on the somatosensory evoked potentials of the nervus 
vagus-how varied stimulation parameters affect VSEP. J Clin Neurophysiol 31(2):143-148

Hagen K, Ehlis A-C, Haeussinger FB, Beeretz S, Kromer GV, Heinzel S, Maetzler W, Eschweiler GW, Berg D, Fallgatter AJ, Metzger FG (2015) The TREND Study Consortium (2015). The relation of SMI and the VSEP in a risk sample for neurodegenerative disorders. J Neural Transm 122(8):1167-1174

Keller J, Bless H, Blomann F, Kleinböhl D (2011) Physiological aspects of flow experiences: skills-demand-compatibility effects on heart rate variability and salivary cortisol. J Exp Soc Psychol 47:849-852

Kraus T, Kiess O, Hösl K, Terekhin P, Kornhuber J, Forster C (2013) CNS BOLD fMRI effects of sham-controlled transcutaneous electrical nerve stimulation in the left outer auditory canal-a pilot study. Brain Stimul 6(5):798-804

Kreuzer PM, Landgrebe M, Husser O, Resch M, Schecklmann M, Geisreiter F et al (2012) Transcutaneous vagus nerve stimulation: retrospective assessment of cardiac safety in a pilot study. Front Psychiatry 3:70-77

Marrosu F, Serra A, Maleci A, Puligheddu M, Biggio G, Piga M (2003) Correlation between $\mathrm{GABA}(\mathrm{A})$ receptor density and vagus nerve stimulation in individuals with drug-resistant partial epilepsy. Epilepsy Res 55:59-70

Murphy PR, O'Connell RG, O'Sullivan M, Robertson IH, Balsters JH (2014) Pupil diameter covaries with BOLD activity in human locus coeruleus. Hum Brain Mapp 35(8):4140-4154

Nemeroff CB, Mayberg HS, Krahl SE, Mcnamara J, Frazer A, Henry TR et al (2006) VNS therapy in treatment-resistant depression: clinical evidence and putative neurobiological mechanisms. Neuropsychopharmacology 31:1345-1355

Peifer C (2012) Psychophysiological correlates of flow-experience. In: Engeser S (ed) Advances in flow research. Springer, New York, pp 139-165

Peifer C, Schulz A, Schächinger H, Baumann N, Antoni CH (2014) The relation of flow-experience and physiological arousal under stress-can u shape it? J Exp Soc Psychol 53:62-69

Peifer C, Schächinger H, Engeser S, Antoni CH (2015) Cortisol effects on flow experience. Psychopharmacology (Berl) 232(6):1165-1173
Peifer C, Lehrich JS, Ingwersen J, Schächinger H, Antoni CH (2017) Difficult but (ir)relevant? Using the demand-skill-fit paradigm to investigate the relationship between flow-experience and stress (under review)

Peuker ET, Filler TJ (2002) The nerve supply of the human auricle. Clin Anat 15:35-37

Raedt R, Clinckers R, Mollet L, Vonck K, El Tahry R, Wyckhuys T et al (2011) Increased hippocampus noradrenaline is a biomarker for efficacy of vagus nerve stimulation in a limbic seizure model. J Neurochem 117:461-469

Roosevelt RW, Smith DC, Clough RW, Jensen RA, Browning RA (2006) Increased extracellular concentrations of norepinephrine in cortex and hippocampus following vagus nerve stimulation in the rat. Brain Res 1119:124-132

Sellaro R, van Leusden JW, Tona KD, Verkuil B, Nieuwenhuis S, Colzato LS (2015) Transcutaneous vagus nerve stimulation (tVNS) enhances post-error slowing. J Cogn Neurosci 27:2126-2132

Sheehan DV, Lecrubier Y, Sheehan KH, Amorim P, Janavs J, Weiller $E$ et al (1998) The mini-international neuropsychiatric interview (M.I.N.I.): the development and validation of a structured diagnostic psychiatric interview for DSM-IV and ICD-10. J Clin Psychiatry 59:22-23

Steenbergen L, Sellaro R, Stock AK, Verkuil B, Beste C, Colzato LS (2015) Transcutaneous vagus nerve stimulation (tVNS) enhances response selection during action cascading processes. Eur Neuropsychopharmacol 25(6):773-778

Tozman T, Magdas ES, MacDougall HG, \&nVollmeyer R (2015) Understanding the psychophysiology of flow: a driving simulator experiment to investigate the relationship between flow and heart rate variability. Comput Hum Behav 52:408-418

van Leusden JW, Sellaro R, Colzato LS (2015) Transcutaneous vagal nerve stimulation (tVNS): a new neuromodulation tool in healthy humans? Front Psychol 6:102

Ventureyra EC (2000) Transcutaneous vagus nerve stimulation for partial onset seizure therapy. Child's Nerv Syst 16(2):101-102 